\title{
STANDARDS FOR TESTING MAGNETIC PERMEAMETERS
}

\author{
By Raymond L. Sanford
}

\section{ABSTRACT}

The testing of magnetic permeameters is greatly facilitated by the use of calibrated test specimens used as standards. The test method employed for calibration must be an absolute one whose accuracy can be estimated without reference to any other method. In order to be suitable for use as magnetic standards, test specimens must conform to certain requirements the most important of which is magnetic uniformity along their length. The paper gives reasons for adopting the Burrows permeameter as the standard method for calibration, shows the effect of nonuniformity in the specimens, and gives the characteristics of four bars which have been found to be sufficiently uniform to warrant their use as magnetic standards.

\section{CONTENTS}

I. Introduction

II. Accuracy of magnetic tests. 178

III. Standard testing method. 179

IV. Standard specimens._. 181

V. Summary

\section{INTRODUCTION}

In recent years magnetic testing has been steadily increasing in importance. Several million dollars worth of materials are now purchased annually on the basis of specifications as to magnetic quality. In order to avoid misunderstandings and disputes between producers and consumers of such materials it is necessary to know the degree of accuracy obtainable with the various kinds of testing apparatus used for checking the quality of materials so purchased.

The designer of electrical machinery is also interested in the accuracy of data on the magnetic properties of materials, since modern methods of control make feasible much closer designs than could previously be made.

Added interest in magnetic testing has resulted from recent developments in the field of magnetic analysis in which the relationships between the magnetic and other physical properties of materials are of fundamental importance. In order to compare the results obtained by various investigators in this field it is essential that the accuracy of the magnetic testing methods employed be definitely established.

For the testing of large numbers of specimens on a routine basis it is no longer satisfactory to employ methods which require the preparation of samples of special form such as toroids or ellipsoids or the winding of separate magnetizing and test coils for each speciman and, consequently, several different types of apparatus have been developed into which samples of simple form are inserted for testing: These are called magnetic permeameters or simply permeameters.

The permeameters in general use at the present time differ more or less radically in principle and it is not always possible to estimate 
the degree of accuracy obtainable from purely theoretical considerations. For this reason the intercomparison of methods is greatly facilitated by the use of carefully selected and prepared test specimens which may properly be considered as magnetic standards. For the calibration of such magnetic standards it is necessary to use a method of known accuracy, preferably an absolute one in the sense that its constants can be determined from its own dimensions and whose accuracy can be estimated without reference to any other method.

In the United States the Burrows permeameter ${ }^{1}$ has been accepted as the standard method for many years. It is an absolute method in the sense indicated above and can be used for straight bars over a wide range of sizes. It is somewhat complicated to operate, however, and requires duplicate specimens, one of which is not tested but used as an auxiliary. Moreover, it is known to be exceedingly sensitive to variation in permeability along the length of the specimen. This constitutes a source of uncertainty such that the degree of accuracy obtained for a nonuniform bar can not be determined.

The Fahy Simplex permeameter ${ }^{2}$ has recently been coming into rather wide use in this country and to some extent abroad. From the standpoint of simplicity and ease of manipulation it has certain advantages over the Burrows method. Another instrument recently developed is the $J$ permeameter ${ }^{3}$ described by B. J. Babbitt of the Western Electric Co. In addition to these methods there are in use, to a more limited extent, various instruments not commercially available, but designed and built by individual testing engineers solely for their own use.

With the growth in importance of magnetic testing the question of the accuracy of the various methods has become more and more important. Intercomparisons previously made, in which the same samples were tested in different permeameters, have not yielded definite and conclusive results and it has become more and more apparent that the difficulty is due primarily to the use of specimens which are not suitable as magnetic standards.

In an investigation undertaken over three years ago for the purpose of checking the accuracy of the Fahy Simplex permeameter it early became evident that before a satisfactory conclusion could be reached it would be necessary to accumulate a set of standards better than any then available and, therefore, first attention was given to this phase of the problem. The following is a report of the investigation on magnetic standards for testing permeameters

\section{ACCURACY OF MAGNETIC TESTS}

In stating the accuracy of magnetic tests it is necessary to take account of the fact that each point on the normal induction curve involves the determination of two quantities, magnetizing force and induction, each one of which may be in error by different amounts. Consequently, the simple statement that a given point is accurate within a certain per cent is not explicit. It is necessary to state either that values of induction corresponding to given values of magnetizing force are accurate within a certain amount, or that

1 Burrows, The Determination of the Magnetic Induction in Straight Bars, B. S. Bull. 6 (Sci. Paper

No. 117), p. 31, 1909.

3 Babbitt, An Improved Permeameter for Testing Magnet Steel, J. Opt. Soc. Am., 17, p. 47; 1928. 
values of magnetizing force corresponding to a given value of induction have a certain degree of accuracy.

On account of the characteristic shape of the normal induction curve neither one of these statements is entirely satisfactory over the whole range. In the steep part of the curve, for instance, a very small variation in the value of the magnetizing force corresponds to a relatively large difference in induction, while at the higher inductions, where the slope of the curve is small, a small change in induction corresponds to a very large variation in magnetizing force. The use of different forms of statement for different parts of the magnetization curve is awkward and cumbersome, however, and it seems advisable to adopt the form to which the least objection can be made.

It is sometimes argued that since the value of induction $B$ can ordinarily be determined to a very high degree of accuracy compared to that attainable in the measurement of the magnetizing force $H$, we should always make the statement in terms of the accuracy of the value of $H$ for a given value of $B$. However, given a pair of corresponding coordinates as experimentally determined we are usually more concerned as to how near the plotted point comes to the true curve than we are with the accuracy of the individual coordinates. It is even conceivable that values of $B$ and $H$, each of which is in error as experimentally determined, might, when plotted, fall exactly on the true curve.

It is obvious that in the upper part of the normal induction curve a 1 per cent variation in $B$ may correspond to a variation in $H$ of 10 per cent or more. It is not so obvious what the ratio may be in the steep part of the curve. Examination of a considerable number of curves for materials of widely different magnetic characteristics reveals the fact that the percentage variation in $B$ rarely exceeds twice that in $H$. It appears most satisfactory, therefore, to state the accuracy of magnetic data by saying that the value of induction corresponding to a given value of magnetizing force is accurate within a given percentage. This form of statement is used throughout the present paper.

In comparison with many other kinds of physical measurements the accuracy attainable in magnetic testing is not high. This is due in large part to the fact there is no known insulator of magnetic flux and consequently there is usually a certain amount of unavoidable magnetic leakage. Such leakage is enhanced by the presence in the magnetic circuit of mechanical joints and air gaps and by variations in permeability in the material making up the circuit. It happens, however, that magnetic material is seldom very uniform and the degree to which the results of tests on individual samples of material represent the properties of the whole lot depends fully as much on the sampling as upon the accuracy of the testing method. For this reason an accuracy of better than 1 per cent is rarely required and from 2 to 5 per cent is usually adequate.

\section{STA̧DARD TESTING METHOD}

The condition that the test method employed for the calibration of magnetic standards shall be an absolute one, whose accuracy can be estimated without reference to any other method, is fully met by the Burrows compensated double-yoke permeameter ${ }^{4}$ provided that 
the test specimens possess the requisite degree of uniformity. Figure 1 shows the magnetic circuit and the relative position of the magnetizing and test coils. The test specimen and its auxiliary, which should be of the same size and material, are joined at the ends by soft iron yokes which make good magnetic joints and complete the magnetic circuit. The magnetizing coils $T$ and $A$ are located over the test rod and auxiliary, respectively. Coil $J$ is in four sections, connected in series, and located over the ends of the rods as near to the joints as possible. In operation the currents in these three windings are so adjusted before each reading that there is equal flux in the two rods and no leakage from the greater part of the test rod. When this condition is realized the value of the applied magnetizing force can be calculated from the current and number of turns per centimeter in the solenoid surrounding the test rod. For testing the compensation and determining the value of the induction when the compensation is properly adjusted there are three test coils designated as $t, a$, and $j$, respectively. These coils are each of the same number of turns and are distributed as shown in the figure; $t$ is wound over

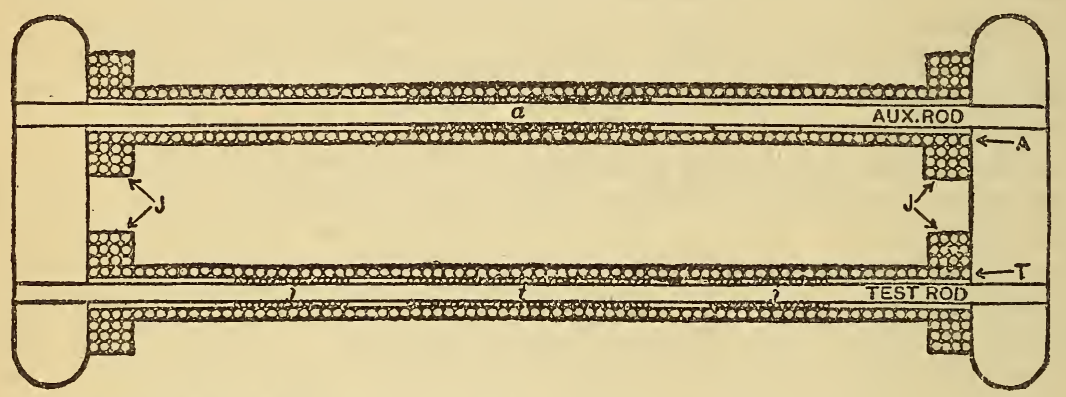

FIgURE 1.-Magnetic circuit of the Burrows permeameter, showing the relative positions of the magnetizing and test coils

the middle of the test bar, $a$ over the middle of the auxiliary bar, and $j$ is wound half over one end and half over the other end of the test bar far enough away from the yokes and joints to avoid disturbances from these causes. When, upon reversal of the current in the magnetizing windings, there is no residual deflection in the ballistic galvanometer whether connected to $t$ and $a$ or to $t$ and $j$ in series opposition, the magnetizing currents are properly adjusted. Under these conditions the induction is measured in terms of the deflection of the ballistic galvanometer connected to $t$ alone and the magnetizing force is proportional to the current in the winding $T$.

It is not difficult to understand why this method is sensitive to the influence of nonuniformity in the test specimen. A balance is indicated when the total flux in the part of the specimen under the test coil $t$ is equal to the average of the values under the two halves of coil $j$. If the specimen is uniform the induction is uniform throughout the length of the bar included between the two halves of the coil $j$ when a balance is indicated. Under this condition there is no leakage from the middle portion of the bar and the magnetizing force can be calculated in terms of the current in the coil $T$.

If the specimen is not uniform, the flux generally does not have the same value under the two halves of coil $j$ when a balance is indicated, 
and, consequently, the induction is not uniform along the length of the bar. The resulting leakage gives rise to magnetizing forces in addition to that due to the current in coil $T$. Under this condition the values obtained are not correct even for the part of the specimen under the coil $t$, neither do they represent average values over the length of the bar included between the two halves of the coil $j$. The errors thus introduced by nonuniformity in the specimen are indeterminate and may be large.

The test coils $t, a$, and $j$ are generally wound on the forms used for the magnetizing windings or on auxiliary forms of only slightly smaller area. If the area of the specimen is small with respect to that of the test coils there is a tendency toward overcompensation; that is, an excessive amount of current is required in the compensating coils at the ends, and, consequently, the normal induction curve as observed will be too high. This has been shown by winding test coils directly on the specimen and comparing the results obtained with these coils with the values determined using the regular test coils. The discrepancy is greater the smaller the specimen.

This difficulty can be overcome by using an arrangement due to Gokhale. ${ }^{5}$ The modification consists in substituting for the test coil $j$ another coil having a large number of turns and which is placed within the magnetizing winding adjacent to but not surrounding the specimen. Its axis is parallel to that of the test bar. When such a test coil is connected to the ballistic galvanometer and the magnetizing current is reversed the galvanometer throw is proportional to the intensity of the magnetic field. With this arrangement the adjustment for compensation consists in setting the compensating current to such a value that the field as indicated by the test coil is equal to that calculated in terms of the current in the magnetizing coil. This is conveniently done by connecting a variable mutual inductance with its primary winding in series with the magnetizing coil $T$ and its secondary in series opposing the test coil. If the mutual inductance is set to equal the value calculated for the magnetizing coil and test coil in terms of the area turns of the test coil and the constant of the magnetizing coil the compensation is correct when there is no residual deflection of the galvanometer upon reversal of the current. With this arrangement it is estimated that, for a specimen of uniform permeability along its length, observed values are accurate to well within 1 per cent.

Several other possible methods were considered for the calibration of magnetic standards, including the magnetometer, the link method of Spooner, ${ }^{6}$ a compensated single yoke method, and the new Picou permeameter. ${ }^{7}$ However, since none of these appeared to have any particular advantage over the Burrows method, and since the limitation as to uniformity of specimen is common to the other absolute methods, the Burrows permeameter was adopted for the calibration of our magnetic standards.

\section{STANDARD SPECIMENS}

There are certain more or less obvious requirements which must be fulfilled for a test specimen to be suitable as a magnetic standard.

6 United States Patent No. 1559085, Oct 27, 1925.

6 Spooner, Methods of Magnetic Testing, Elec. J., 18, p. 351; 1921.

7 Picou, Nouveau Perméamètre de la Société des Atêliers, J. Carpentier, Rev. Gén. de l'Elec., 20, p. $346,1926$. 
The specimen should be of uniform dimensions and straight. If the bar is not straight it will be strained when clamped in the testing apparatus and it is well known that a relatively slight amount of mechanical strain may alter the magnetic properties by a considerable amount. It is also desirable that the specimens be metallurgically stable. Under certain conditions, changes in internal structure or strain may proceed at a slow rate over a considerable period of time even at ordinary temperatures. Such changes bring about corresponding changes in magnetic properties and should be completed by a suitable aging process before the specimens are used as magnetic standards.

By far the most important requirement is that of magnetic uniformity along the length of the specimen. Although Burrows in his

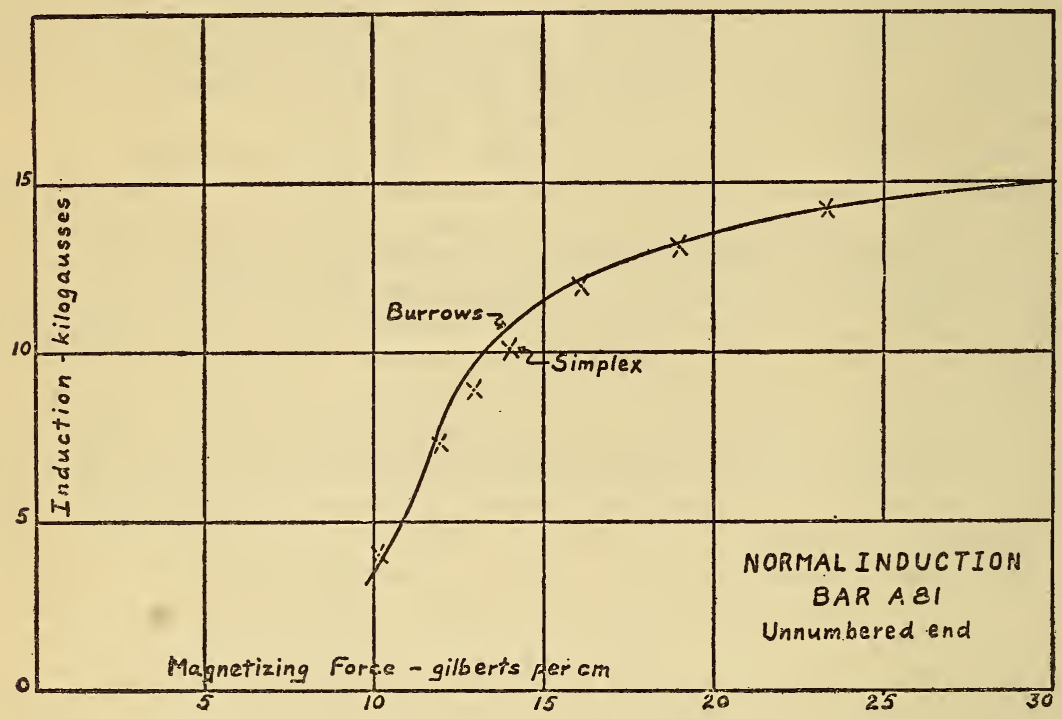

FIgURe 2.-Normal induction curve for unnumbered end of bar A81

In this position in the Burrows permeameter a magnetically hard spot is directly under one of the $j$ test coils.

original article describing the compensated double-yoke method ${ }^{8}$ called attention to this point it is often disregarded, and differences in results obtained on the same specimen by differen $\iota$ methods, one of which is accepted as standard, are interpreted as errors. This is not a safe practice. Such conclusions are never justified unless the test specimens are known to possess the requisite degree of uniformity.

Figures 2 and 3 are illustrative of the different results which might be obtained if nonuniform bars are used as standards. The data were all taken on the same bar, No. A81. This bar is $32.5 \mathrm{~cm}$ long. The Burrows permeameter coils used require a length of only $25 \mathrm{~cm}$, so that there was a total projection of $7.5 \mathrm{~cm}$. The two curves give a comparison of the indications obtained with the Fahy Simplex permeameter with those of the Burrows permeameter. It is evident that quite different conclusions are arrived at if the bar is tested with

${ }^{8}$ Burrows, The Determination of the Magnetic Induction in Straight Bars, B. S. Bull. 6 (Sci. Paper No. 117), p. 31; 1909 . 
the numbered end flush with the yokes from those to be drawn from tests made with the other end flush. If we should assume, for instance, that the Burrows results are accurate, we would conclude on the basis of Figure 2 that the Simplex results are low in the region of the "knee" of the curve, the maximum difference being as much as 10 per cent. On the other hand, if we accept the evidence of Figure 3, we would say that the Simplex gives results that are too high in the range of magnetizing force up to 30 gilberts per $\mathrm{cm}$ with a maximum error greater than 20 per cent. Obviously, these conclusions can not both beright and since the position of the bar in the apparatus was the only variableit is evident that lack of uniformity in the bar must have been responsible for the difference.

In 1916 the author described a method ${ }^{9}$ for determining the degree of uniformity of magnetic test specimens. This method con-

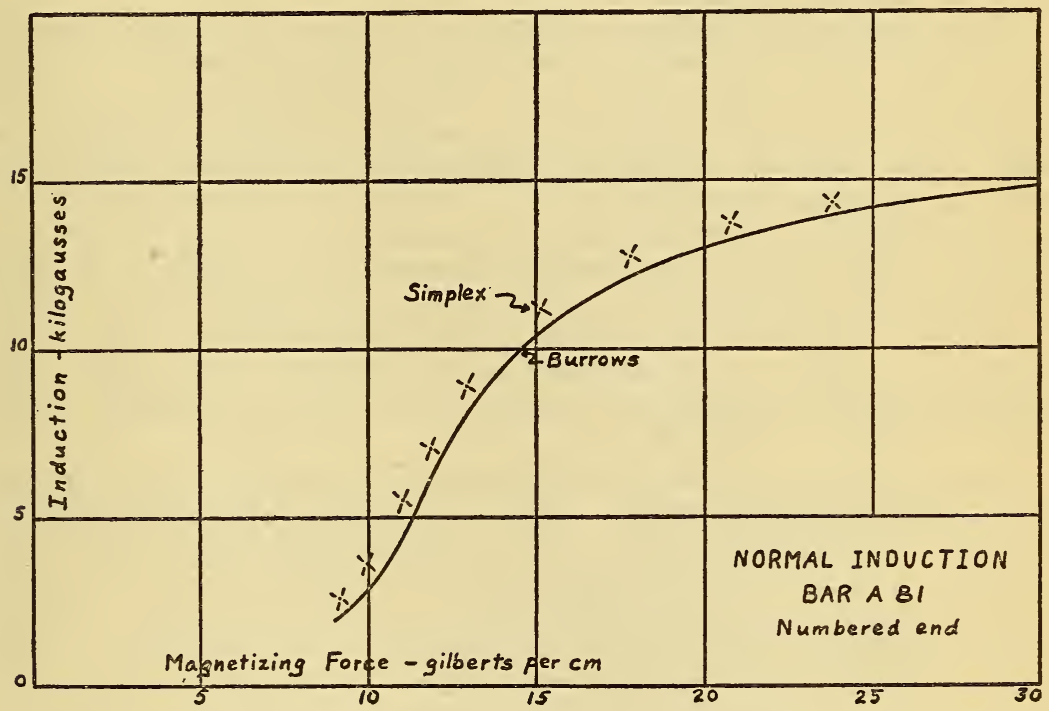

Figdre 3.-Normal induction curve for numbered end of bar A81 In this position in the Burrows permeameter a magnetically hard spot is directly under the middle
test coil.

sists in measuring the magnetic leakage along the length of a specimen when magnetized between the poles of a suitable electromagnet. It has been found that for a uniform specimen the rate of variation of leakage along the length is uniform except for portions very near the ends. By plotting the rate of variation of leakage, therefore, a curve is obtained which for a uniform specimen is straight and horizontal. Deviations indicate lack of magnetic uniformity. Curves so plotted have been called uniformity curves. This method, although giving a good qualitative idea of the uniformity of a given specimen, does not yield quantitative results because the specimen so magnetized is not subjected to a uniform value of magnetizing force throughout its whole length. It would be very desirable to be able to specify the degree of uniformity on a percentage basis and, there-

' Sanford, Determination of the Degree of Uniformity of Bars for Magnetic Standards, B. S. Bull. 14, (Sci. Paper No. 295), p. 1, 1916. 
fore, an attempt was made to discover a way in which this could be done.

Three methods were investigated; measurements with the Burrows. apparatus on specimens long enough so that different portions can be included between the yokes, a miniature Fahy Simplex permeameter having a span of only $5 \mathrm{~cm}$. and a special yoke of $\mathrm{H}$ form, by means of which successive portions of the test bar can be compared with a fixed standard.

Both the Burrows apparatus and the small Simplex permeameter include in the test so long a section of the specimen that the averaging of permeability over this section masks actual variations along the bar. It was found also that reproducible results could not be obtained with the yoke apparatus, probably on account of variability in the contact reluctance. It seemed best, therefore, to continue the use of the leakage method.

It was found in the earlier investigation that for 1 per cent accuracy no ordinate of the uniformity curve may depart from the average value by more than 10 per cent. In order to characterize the various specimens examined for uniformity a so-called uniformity factor was used. This factor is found by subtracting the largest percentage deviation from the mean from 100 . On this basis no specimen having a uniformity factor less than 90 would be suitable to use as a standard. It should be understood, however, that there is no definite relationship between this factor and the degree of accuracy obtainable in the determination of the normal induction curve because the effect of nonuniformities depends not only upon their magnitude but also upon their distribution along the length of the bar.

During the course of the investigation a large number of bars have been examined for uniformity. These specimens came from a number of sources. Some were sent to the bureau for test, some were taken from the stock of steel maintained for the use of the bureau instrument shop, and some were taken from special lots of steel acquired by the bureau for use in various metallurgical investigations. After a large number of bars had been examined and found unsuitable for the purpose it was finally decided to attempt to obtain standards by subjecting a series of specimens to heat treatments designed to produce the greatest uniformity of structure, and grinding them to size under water in order to avoid localized heating. Great care was also taken to see that at no stage in their preparation were the bars bent or subjected to mechanical strain which could produce permanent effects. These bars were of plain carbon steel having various carbon contents so as to cover as wide a range of permeability as possible.

Typical results obtained by the leakage methods are shown by the uniformity curves illustrated in Figure 4 . Out of the 14 bars in this series only $2, A 21$ and $A 22$, were found to be uniform within the required limits. Bar A21, with a uniformity factor of 94 , was chosen to be used as a standard, with A22, having a factor of 92 , as an auxiliary for use in the Burrows permeameter. Some of the other bars, while not suitable for standards, were tested to show the effects of nonuniformity on the indications of the Burrows permeameter.

The uniformity curve for bar A81 explains the results shown in Figures 2 and 3 . The data for Figure 3 were taken with the magnetically hard spot directly within the middle test coil. When the observations for Figure 2 were taken the hard spot was under one of 
the $i$ test coils. The maximum difference in the induction corresponding to a given magnetizing force is of the order of 20 per cent. Obviously this specimen is not suitable to be taken as a magnetic standard. This is not an isolated or unusual case, but is typical of the results obtained on scores of bars examined during the course of the investigation and is cited to bring out very clearly how unsafe it is to draw conclusions as to the accuracy of a given method from results obtained with a specimen not previously checked for uniformity.

Bar A61 does not show sharp variations, but is magnetically softer in the middle than at the ends. The uniformity factor is much

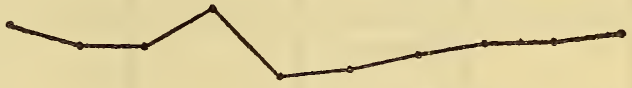

Bar $A 8 /$ UF 64
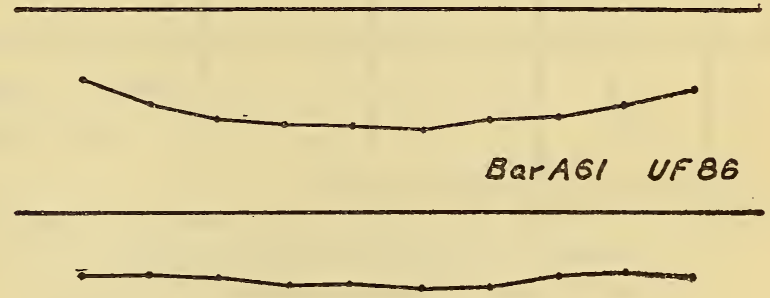

BarA2I UF 94

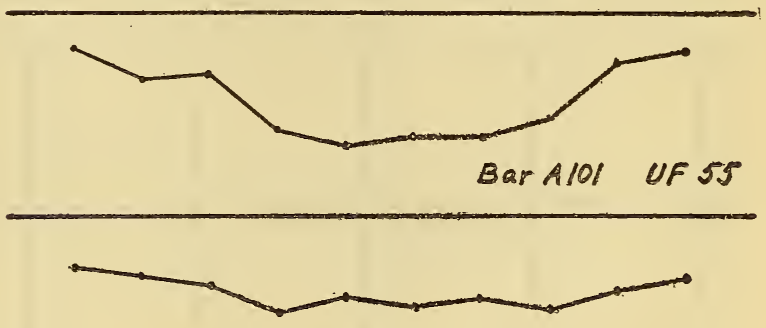

Bar A103 UF 89

FIGURE 4.-Uniformity curves for five of the bars examined by the leakage method

better than for bar A81, being 86 as against 64 for A81. The differences obtained from a test at the middle of and one with one end flush with the yokes, as indicated in Figure 5, are much less than for bar A81. The greatest difference in induction for a given magnetizing force for different portions of the bar is of the order of 10 per cent, whereas for bar A81 it was 20 per cent. Similar results were obtained for other specimens.

Figure 6 shows the results for bar A21. Two sets of observations were taken, one at the middle of the bar, and the other with one end flush. In this case no difference was found greater than the probable experimental error. This is further evidence that A21 is suitable for use as a standard. 
In view of the results of the attempt to produce standard bars by special heat treatment the best course appears to be to continue the

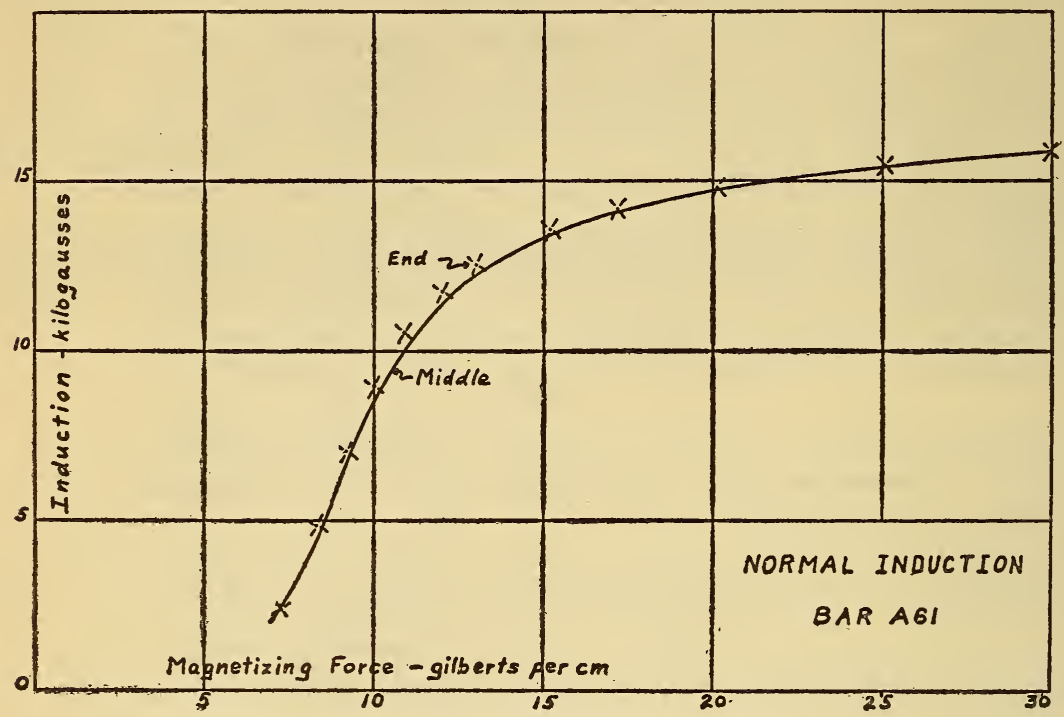

FIgURe 5.-Normal induction for bar $A 61$

This bar is magnetically softer at the middle than at the ends.

examination for uniformity of such test bars as may become available. Since the work on the special series reported above was completed

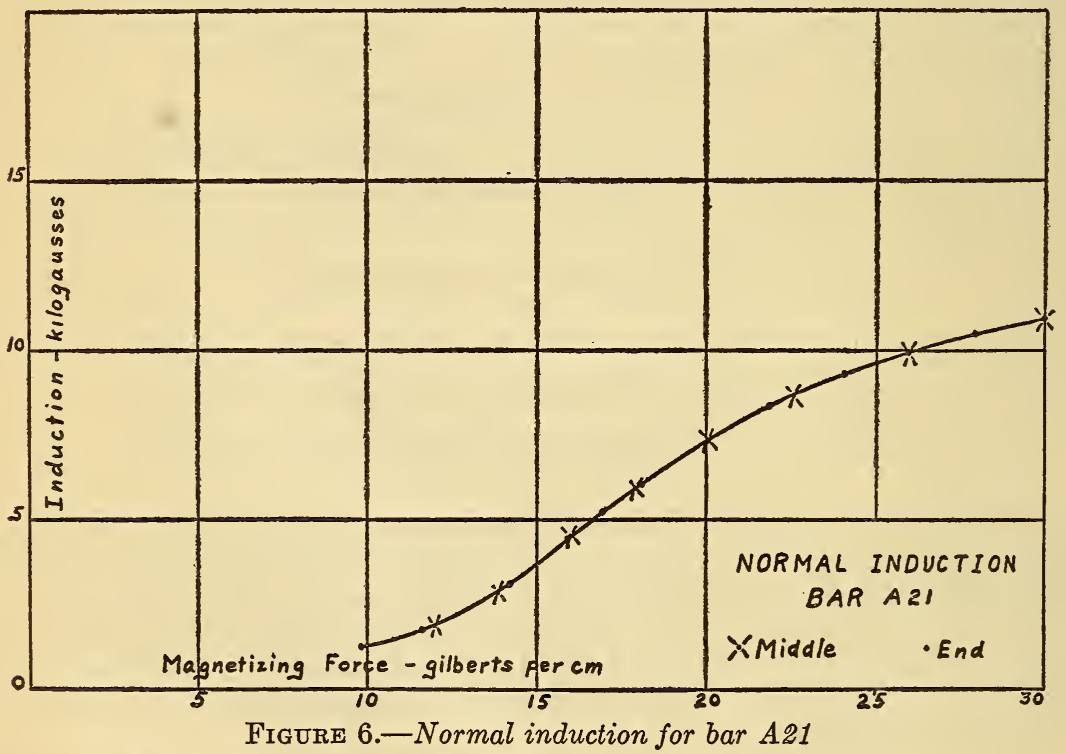

This bar is magnetically sufficiently uniform to serve as a magnetic standard.

three other standards have been accumulated. One is a bar of coldrolled machinery steel which was annealed in order to obtain a relatively high permeability, another is a bar of similar material in the 
"as received" condition, and the third is a bar of tool steel which has not been hardened. All of these specimens are of unknown compo-

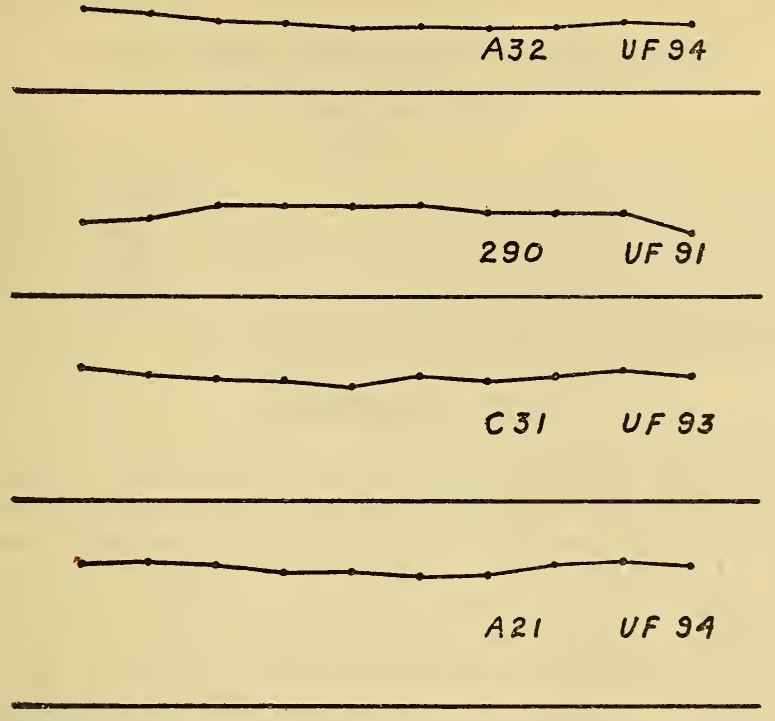

FIGURE 7.-Uniformity curves for four standard bars

sition, but this is not important as we are interested only in their magnetic characteristics. In Figure 7 are shown the uniformity

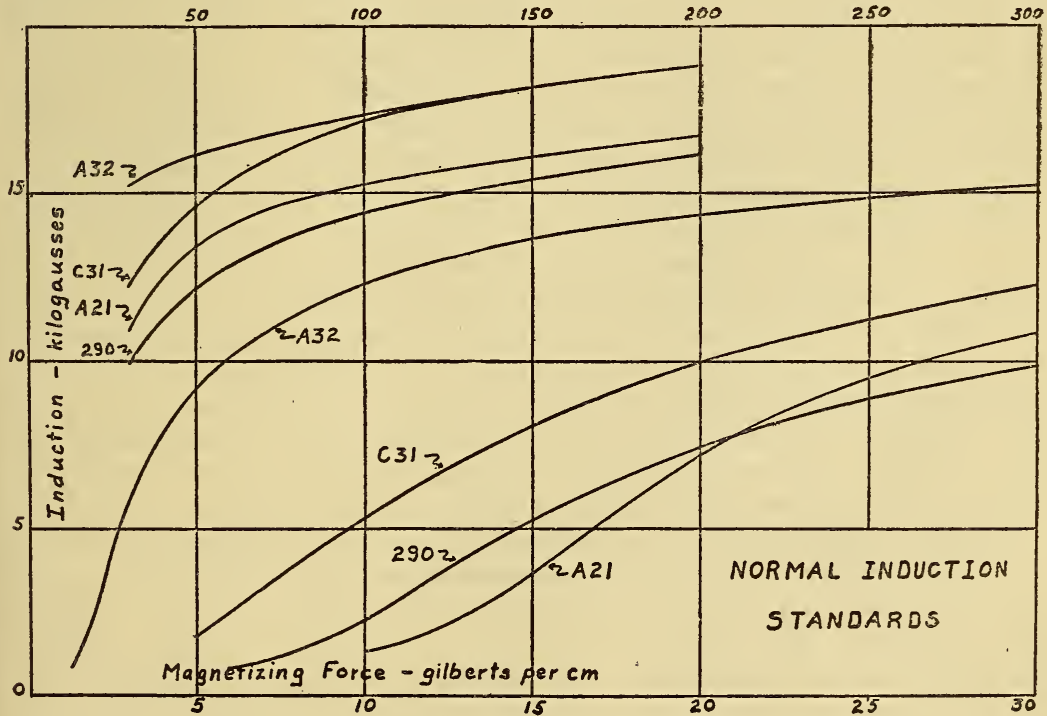

FIGURE 8.-Normal induction curves for four standard bars showing range in properties covered

curves for the four standard bars which have been selected up to date, and Figure 8 shows the normal induction. It can be seen that 
a fair range of permeability is covered, but other bars will be added to this collection as suitable ones are discovered.

Hysteresis data for the four standards are given in Table 1.

TABLE 1.-Hysteresis data for magnetic standards

[Maximum magnetizing force, 200]

\begin{tabular}{|c|c|c|c|}
\hline No. & $\left|\begin{array}{l}\text { Maximum } \\
\text { induction }\end{array}\right|$ & $\begin{array}{l}\text { Residual } \\
\text { induction }\end{array}$ & $\begin{array}{l}\text { Coercive } \\
\text { force }\end{array}$ \\
\hline $\begin{array}{l}\text { A } 32{ }^{2} \\
\text { C290 }\end{array}$ & $\begin{array}{l}18.83 \\
18.99 \\
16.09 \\
16.70\end{array}$ & $\begin{array}{r}\text { 10. } 70 \\
7.60 \\
8.75 \\
12.25\end{array}$ & $\begin{array}{r}2.6 \\
\text { 7. } 2 \\
12.5 \\
16.6\end{array}$ \\
\hline
\end{tabular}

Magnetizing forces, in gilberts per $\mathrm{cm}$; induction, in kilogausses.

\section{SUMMARY}

In view of the present importance of magnetic testing, and the desirability of establishing the accuracy of various magnetic permeameters now in use, an investigation has been carried out on magnetic standards. It is not always possible to estimate the accuracy of a given magnetic testing method from purely theoretical considerations. The testing of magnetic permeameters is greatly facilitated, therefore, by the use of carefully chosen specimens calibrated by a standard testing method. Such specimens may properly be considered as magnetic standards.

In the course of the investigation the principal points considered were the choice of the standard testing method and the requirements which test specimens must fulfill in order to serve satisfactorily as magnetic standards. The Burrows compensated double-yolk permeameter, which has long been accepted as standard in the United. States, fulfills the necessary requirements that its constants can be determined from its own dimensions and its accuracy can be estimated without reference to any other test method. None of the other methods considered appear to have any advantage over the Burrows permeameter and consequently it was adopted for the calibration of our standards.

The principal requirement, as well as the most difficult one, to meet in order that a specimen shall be suitable for use as a standard, is that it shall be magnetically uniform along its length. Many bars examined for uniformity by the leakage method previously described were found to be unsuitable for the purpose. The specimens examined included a number that were specially chosen and heat treated with the idea of producing the most homogeneous structure possible. Several of these nonuniform bars were tested in the Burrows apparatus with different sections included between the yokes in order to demonstrate how unsafe it is to draw definite conclusions as to the accuracy of a given permeameter from the results of tests with nonuniform bars. Four specimens were finally discovered which possess the requisite degree of uniformity. These bars give the same results when different sections of them are tested in the Burrows permeameter, and, consequently, have been chosen for use as magnetic standards. These standards cover a fair range of magnetic permeability, but additional bars will be added to the set whenever suitable ones are discovered.

Washington, September 30, 1929. 